\title{
ALGORITMOS PARA REALCE DE LA ONDA R DEL ELECTROCARDIOGRAMA ${ }^{1}$
}

\author{
Javier E. González Barajas² \\ Cristian C. Velandia Cardenas ${ }^{3}$ \\ Johann A. Nieto Camacho4
}

\section{Prensentación}

El procesamiento digital de señales ha realizado aportes para depurar e identificar características fundamentales de series de tiempo que provienen de diversos fenómenos naturales. Un fenómeno natural, que ha sido el centro de diversas investigaciones, es el comportamiento eléctrico del corazón humano. Este fenómeno es descrito por una señal eléctrica denominada el electrocardiograma (ECG).

$\mathrm{Al}$ aplicar diferentes tecnicas de tratamiento de señales es posible acentuar ciertas caracteristicas presentes en el ECG que son la base para el cálculo de la señal de ritmo cardíaco. El objetivo de este capítulo es presentar los resultados obtenidos en el desarrollo de métodos básicos que acentuan la onda $\mathrm{R}$ presente en el electrocardiograma con el fin de realizar el cálculo de la frecuencia cardíaca instantánea a través de la señal de ritmo cardíaco.

Durante el desarrollo del texto se encontrarán una serie de conceptos que hacen posible entender cómo se generan dichas señales de forma natural en el cuerpo humano, cómo se pueden tratar y posteriormente procesar su información para obtener parametros que ayudan a médicos y a demás profesionales de la salud a diagnosticar enfermedades y médicar pacientes.

1 Proyecto "Sistema digital para la cuantificación eficaz de la frecuencia cardiaca instantánea fase II" perteneciente al semillero de Procesamiento Digital de Señales de la Facultad de Ingeniería Electrónica.

2 Magister en Ingeniería, Docente de la Facultad de Ingeniería Electrónica, División de Ingenierías, Universidad Santo Tomás, Bogotá-Colombia, javiergonzalezb@usantotomas.edu.co

3 Ingeniero electrónico, División de Ingenierías, Facultad de Ingeniería Electrónica, Universidad Santo Tomás, Bogotá-Colombia, cristianvelandia@usantotomas.edu.co

4 Ingeniero electrónico, Facultad de Ingeniería Electrónica, División de Ingenierías.Universidad Santo Tomás, Bogotá-Colombia, johannnieto@usantotomas.edu.co 
Algoritmo para realce de la onda $\mathrm{R}$ del electrocardiograma

El siguiente capítulo presenta las bases teóricas y aportes de algoritmos desarrollados por el Semillero de Investigación en Procesamiento Digital de Señales durante la ejecución preliminar del proyecto: "Sistema digital para la cuantificación eficaz de la frecuencia cardiaca instantánea fase II", aprobado por la convocatoria FODEIN 2014- 2015. 
$\mid$ Introducción|

El electrocardiograma (ECG) es una señal que se caracteriza, en el dominio del tiempo, por tener una amplitud muy reducida ( $1 \mathrm{mv}$ pico a pico aproximadamente) y componentes espectrales entre 0.5 y $100 \mathrm{~Hz}$. Para poder adquirir esta señal es necesario contar con un sistema de acondicionamiento de señales, con la finalidad de poder amplificarlo y filtrarlo para obtener los adecuados valores de amplitud que sean útiles para una posterior etapa de conversión análoga a digital. El proceso de conversión análoga a digital ofrece la oportunidad de obtener una versión discreta de la señal ECG. Esto ofrece facilidades para implementar estrategias de filtrado digital para minimizar las distorsiones causadas por las fuentes de ruido.

Al obtener una señal ECG libre de distorsiones se pueden apreciar diferentes formas de ondas nombradas de la siguiente manera, en orden de ocurrencia: Onda P, Q, R, S y T. La onda R se destaca por ser el punto de referencia para el cálculo de la frecuencia instantánea del electrocardiograma.

Por medio del diseño e implementación de algoritmos se puede calcular la señal de ritmo cardíaco. Esta señal está compuesta por el tiempo entre cada una de las ondas R del electrocardiograma y haciendo uso de ella se obtiene la denominada frecuencia cardíaca instantánea, parámetro de estudio para los profesionales de la salud. Con este parámetro pueden identificar anomalías en los sistemas nervioso y cardiovascular. 


\section{$\mid$ Marco Térico $\mid$}

El electrocardiograma (ECG) es la señal que permite describir el comportamiento eléctrico del corazón. Esta señal está compuesta por una adición de ondas que conforman el complejo cardiaco: onda P, Onda Q, Onda R, Onda S y Onda T. La figura 1 contiene un ejemplo de un electrocardiograma y las diferentes ondas que lo componen.

Figura 1. El electrocardiograma (ECG)

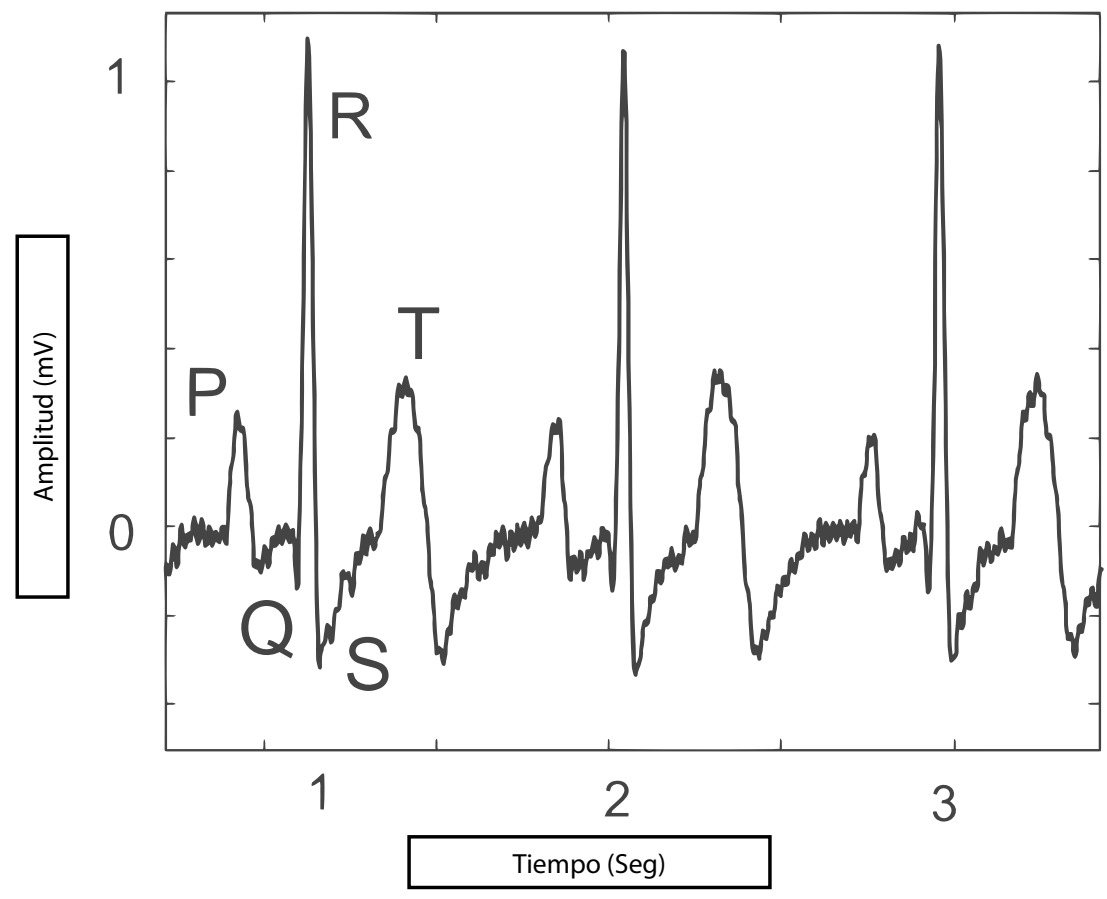

Fuente: elaboración propia 


\section{Escenarios de la formación investigativa}

La onda P está producida por la propagación de la despolarización en las aurículas, y es seguida por la contracción auricular, que produce una ligera elevación de la curva de presión auricular inmediatamente después de la onda P electrocardiográfica.

Aproximadamente 0,16 segundos después del inicio de la onda P, las ondas QRS aparecen como consecuencia de la despolarización eléctrica de los ventrículos, que inicia la contracción de los ventrículos y hace que comience a elevarse la presión ventricular. Por tanto, el complejo QRS comienza un poco antes del inicio de la sístole ventricular.

Finalmente, en el electrocardiograma se observa la onda $T$ ventricular, que representa la fase de repolarización de los ventrículos, cuando las fibras del músculo ventricular comienzan a relajarse. Por tanto, la onda $T$ se produce un poco antes del final de la contracción ventricular (Guyton y Hall, 2011).

Figura 2. Sistema eléctrico del corazón

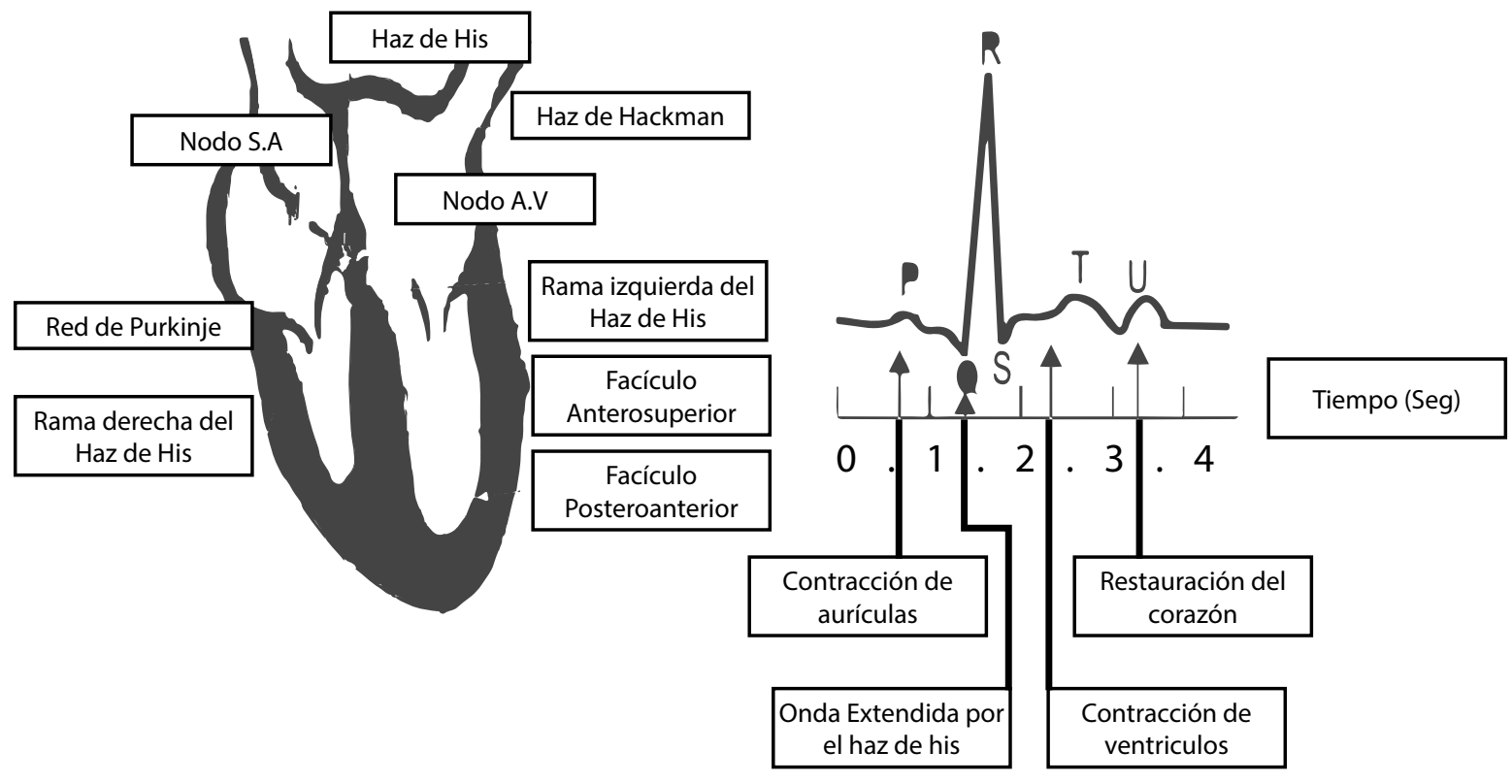

Fuente: elaboración propia

La actividad eléctrica del nodo sinoauricular (sinusal), nodo auriculoventricular (AV), el Haz de His y sus ramas no es registrada en el electrocardiograma, pero mediante el análisis de la secuencia de la propagación del impulso se hace factible la deducción del comportamiento electrofisiológico de dichas estructuras. La figura 2 permite observar la relación de cada onda del complejo 
cardiaco con los componentes del sistema eléctrico del corazón (Koeppen y Stanton, 2009, p. 292).

\section{Acondicionamiento de señales}

El principal componente de un sistema de acondicionamiento para señales electrocardiográficas es el amplificador de instrumentación. Este dispositivo, ya posicionado en el mercado, está compuesto por un arreglo de amplificadores operacionales que proporcionan una ganancia en modo diferencial cuya función es suprimir el ruido presente en ambas señales lo que lo vuelve mucho más sensible dando la oportunidad de trabajar con señales muy pequeñas. La figura 3 contiene un esquema típico de un amplificador de instrumentación.

Figura 3. El amplificador de instrumentación

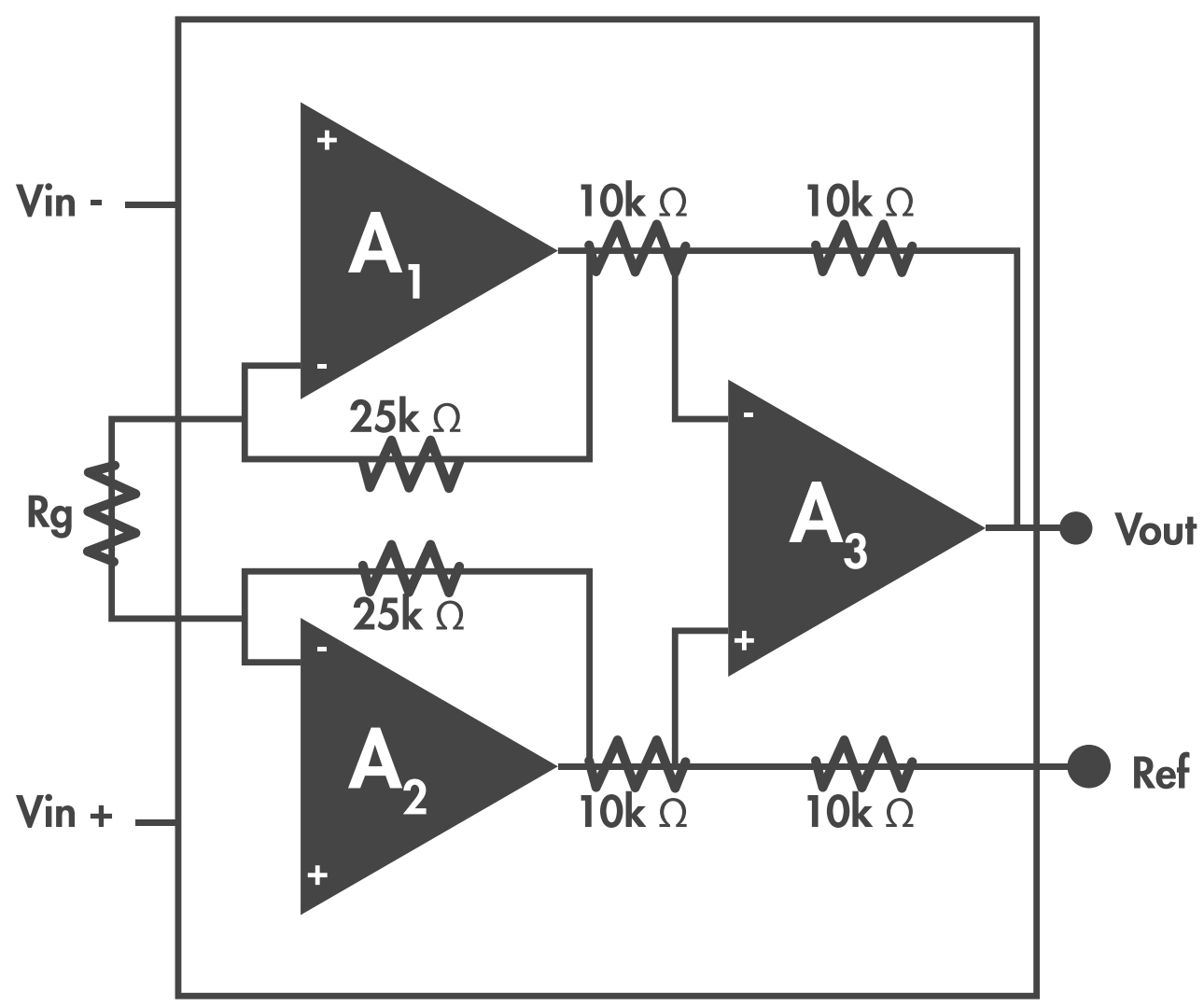

Fuente: elaboración propia 
Las entradas del amplificador de instrumentación (Vin + y Vin-) son conectadas a los electrodos que están posicionados sobre el cuerpo del paciente. Según la posición de los electrodos, se pueden obtener diferentes configuraciones para adquirir la señal ECG. Estas configuraciones se denominan: Derivaciones. La figura 4 ilustra las derivaciones más comunes para un sistema básico de adquisición del ECG.

Figura 4. Configuración según la posición de los electrodos

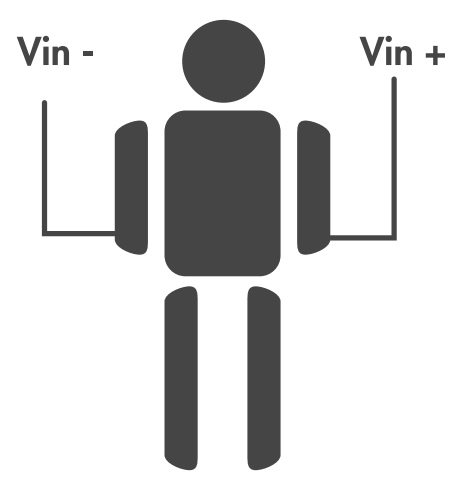

Derivación I

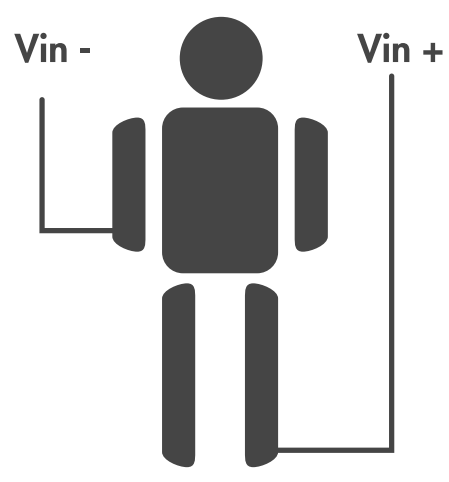

Derivación II

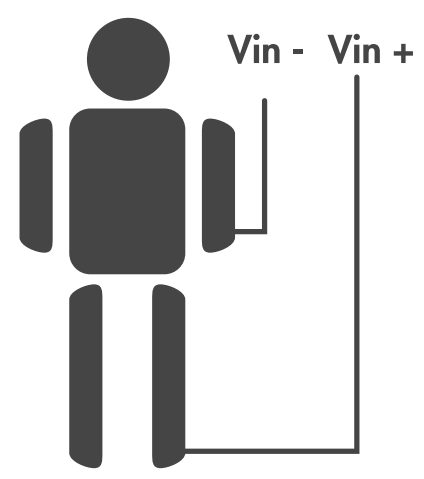

Derivación III

Fuente: elaboración propia

Las derivaciones DI, DII y DII dependen de la posición de los electrodos conectados a las extremidades del paciente: brazo derecho ( $\mathrm{Ra})$, brazo izquierdo (La) y pierna izquierda (LL). La pierna derecha (RL) no posee un electrodo conectado al amplificador de instrumentación. Esta extremidad tiene un electrodo conectado a un circuito de retroalimentación que cumple la labor de conducir una pequeña cantidad de corriente igual a las corrientes de desplazamiento que fluyen en el cuerpo hacia la pierna, el cual es el punto de suma en el lazo de realimentación de tal manera, que el efecto es disminuir el potencial de modo común a un valor muy bajo (Enderle y Blanchard, 2000). 
Figura 5. Circuito manejador de pierna derecha

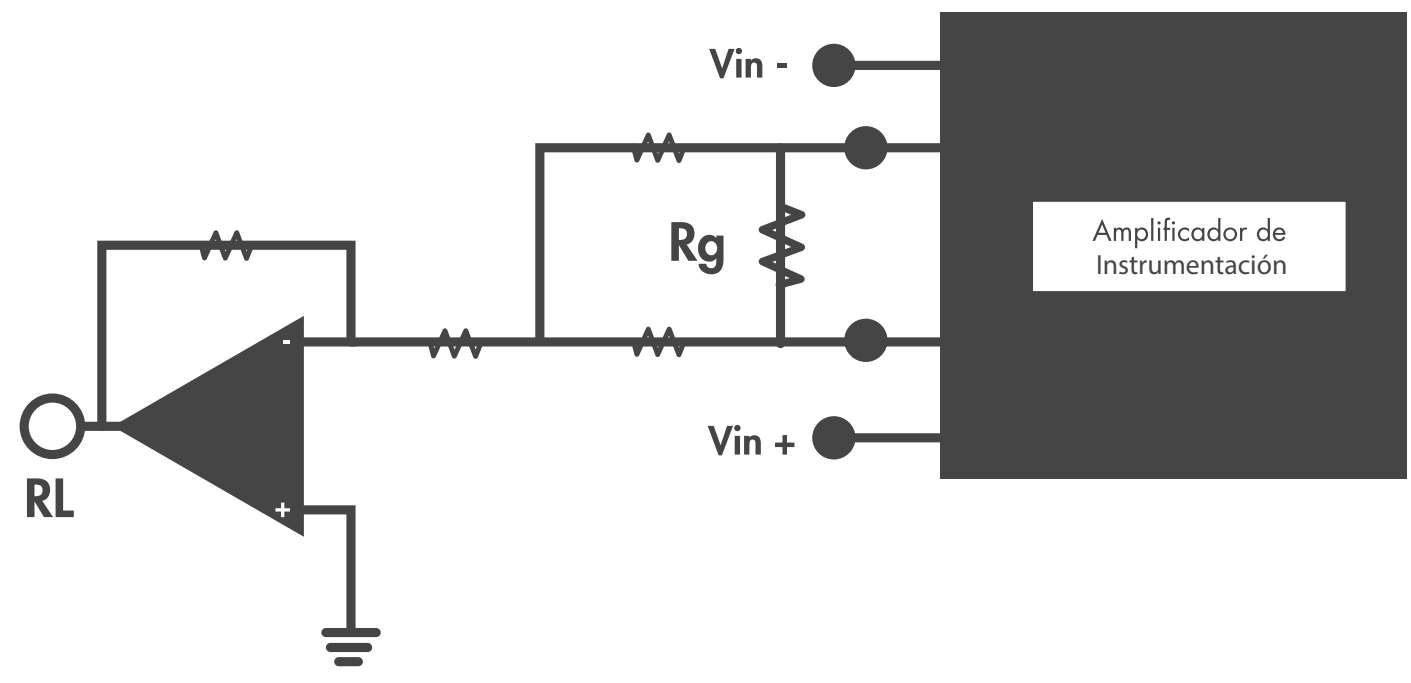

Fuente: elaboración propia

El circuito manejador de pierna derecha, junto con el circuito amplificador de instrumentación se adiciona a un sistema completo de acondicionamiento para la adquisición de la señal ECG. La figura 6 ilustra un diagrama de bloques de un sistema de acondicionamiento de señales.

Figura 6. Sistema de acondicionamiento de señales ECG

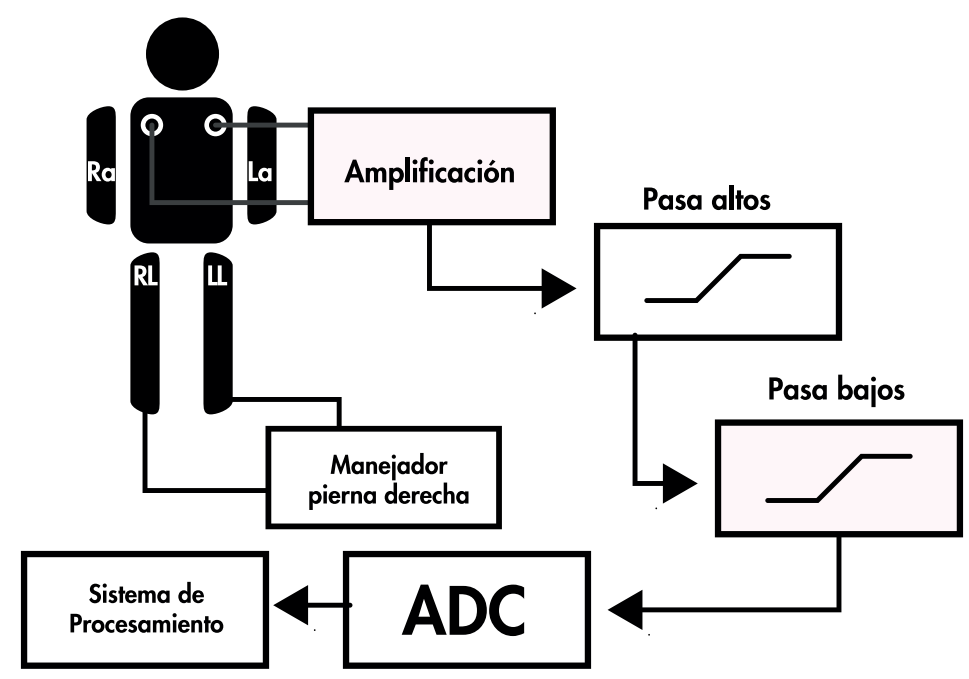

Fuente: elaboración propia 
El sistema de acondicionamiento de señales apropiado para la adquisición del ECG (figura 6) consta de un amplificador de instrumentación que proporciona una ganancia a la diferencia de potencial entre dos electrodos colocados sobre el paciente. Posteriormente se cuenta con una etapa de filtrado analógico compuesta por un filtro pasa altas (frecuencia de corte $=0.5-0.7 \mathrm{~Hz}$ ) y un filtro pasa bajas (frecuencia de corte $=100 \mathrm{~Hz}$ ) (Barrero, González y Pinto, 2011). La Señal ECG puede ser distorsionada por ruidos provocados por las líneas de alimentación de energía eléctrica y movimientos musculares, por lo cual se han generado estrategias basadas en filtros digitales para disminuir sus efectos (González-Barajas y Montenegro, 2014c).

\section{Cálculo de la frecuencia cardíaca instantánea}

La onda $\mathrm{R}$ corresponde al valor positivo de mayor magnitud que se encuentra en el registro electrocardiográfico. Esta onda está precedida por la onda Q y seguidamente aparece la onda $\mathrm{S}$. La detección de la onda $\mathrm{R}$ es un proceso que permite la cuantificación de la frecuencia cardiaca instantánea y es la base de diferentes tipos de análisis del sistema cardiovascular y nervioso autónomo (López y Fernández, 2008).

\section{- Método de banco de filtros para detección de la onda R}

La estructura principal de un banco de filtros se conforma por una señal de entrada $\mathrm{x}(\mathrm{n})$ que es tratada por una serie de filtros con respuesta al impulso $\mathrm{Hn}(\mathrm{n})$. Esta estructura genera como salida, diferentes versiones de la señal de entrada $\mathrm{xn}(\mathrm{n})$. La figura 7 ilustra un diagrama de bloques de un sistema de banco de filtros.

Figura 7. Estructura de un banco de filtros

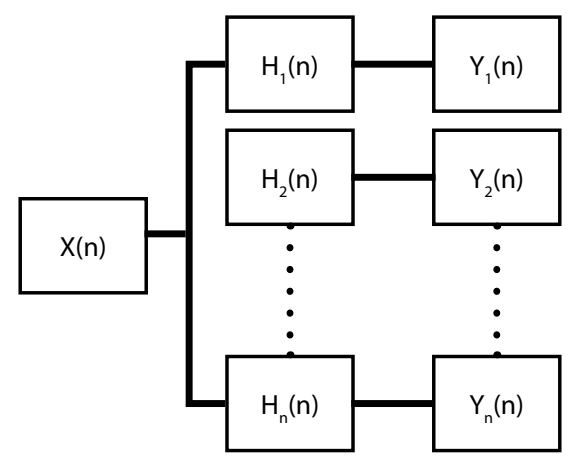

Fuente: elaboración propia 


\section{Algoritmo para realce de la onda $\mathrm{R}$ del electrocardiograma}

Para analizar el electrocardiograma es necesario conocer su rango en frecuencias aproximado, el cual es de $0.1 \mathrm{a} 100 \mathrm{~Hz}$, teniendo esto en cuenta se procede a hacer divisiones en frecuencia aplicando un banco de filtros pasabanda, los cuales van a acentuar las características de la señal en los rangos seleccionados.

A través de las siguientes líneas de código en Matlab se puede implementar un banco de filtros pasabanda:

1. Establecer el rango de frecuencias:

$F=[0.110 ; 1020 ; 2030 ; 3040]$;

$w n=F /(F s / 2)$;

2. Generar la familia de funciones $H k(n)$ :

$$
\begin{aligned}
& \text { fori }=1: 4 \\
& H(i, .)=f i r 1\left(50, w n(i, .),{ }^{\prime}\right. \text { 'bandpass'); } \\
& \text { end }
\end{aligned}
$$

3. Para ejecutar el banco de filtros se implementa la siguiente rutina:

$$
\begin{aligned}
& \text { for } n=1: 4 \\
& X N(n,:)=f i l t e r(H(n,:),[1], x) \text {; } \\
& \text { end }
\end{aligned}
$$

El resultado obtenido en el proceso está almacenado en la matriz XN. Cada fila de esta matriz contiene las diferentes versiones de la señal x(n) de entrada.

La figura 8 contiene el resultado obtenido, en donde se puede observar que la onda R se destaca en la banda entre 10 y $20 \mathrm{~Hz}$. Este resultado permite determinar el rango de frecuencias de las componentes espectrales que están asociadas a la onda R del complejo cardiaco (Tompkins, 1993).

El resultado obtenido en la figura 8 fue el soporte para la generación de publicaciones relacionadas con la detección de la onda R. En ésta publicación el resultado obtenido en la figura 8 permitió establecer el criterio para el diseño de filtros pasa banda en plataformas informáticas y ser la base para implementaciones de algoritmos de umbralización de la onda R (González-Barajas, 2014a). 
Escenarios de la formación investigativa

Figura 8. Señal ECG procesada a través de un banco de filtros

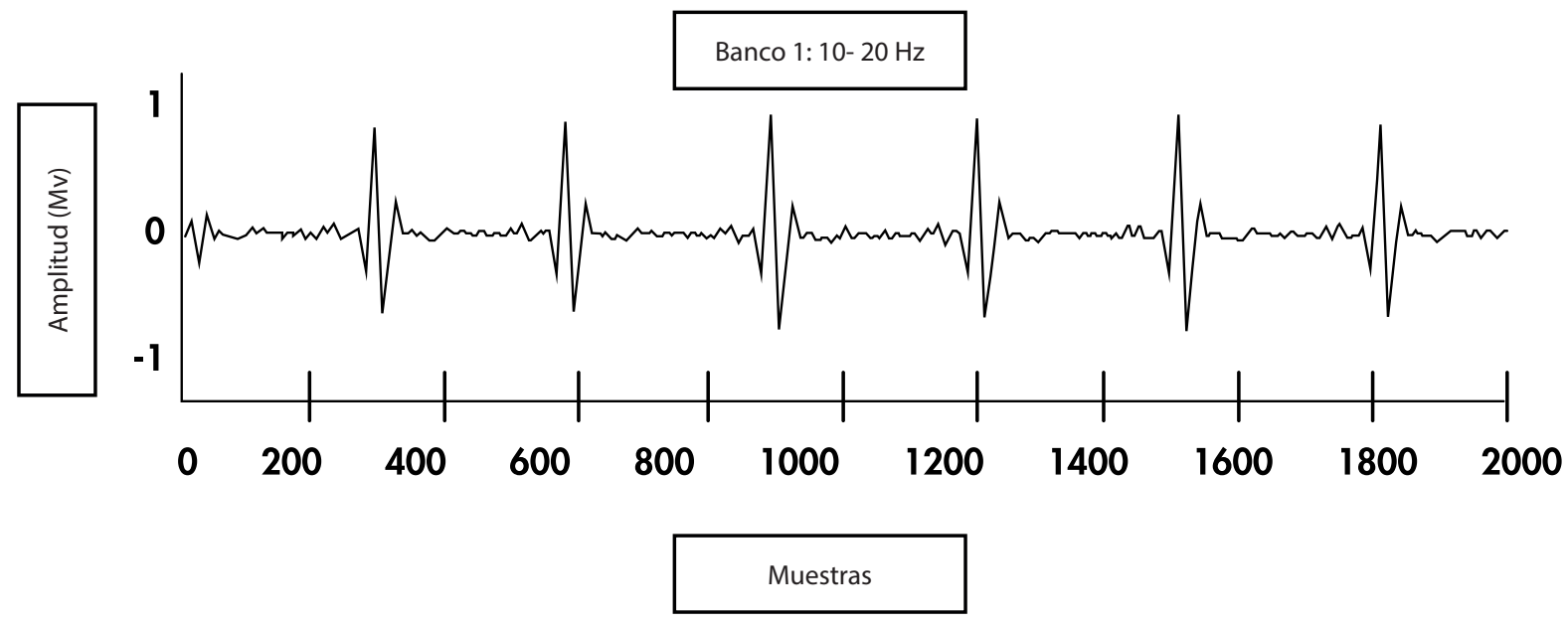

Fuente: elaboración propia

La figura 8 también ha generado aportes en cuanto al diseño de filtros en tiempo real implementado en plataformas basadas en micro controlador. Al establecer un criterio con los rangos de frecuencia que contienen la información de la onda $\mathrm{R}$, se han podido diseñar filtros en tiempo real para el realce de la onda $\mathrm{R}$ (González-Barajas, Velandia-Cardenas y Nieto-Camacho, 2015).

\section{- La Transformada Wavelet Continua}

La Transformada Wavelet Continua está basada en el estudio de señales por medio de un proceso de descomposición. En este proceso, la señal bajo estudio es comparada con una función base denominada Wavelet. Esta estrategia ha sido utilizada para la detección de formas de ondas particulares en contextos como la señal electrocardiográfica y las señales de potencia eléctrica (González-Barajas, Velandia-Cardenas y Nieto-Camacho, 2015).

Una de las funciones wavelets más conocidas es la función Sombrero Mexicano. Esta forma de onda se describe en la ecuación 1

$$
\varphi(t)=\left(1-t^{2}\right) e-\frac{t^{Z}}{z}
$$

La Figura 9 ilustra la representación en el dominio del espacio de la función Sombrero Mexicano. 
Figura 9. Función Sombrero Mexicano

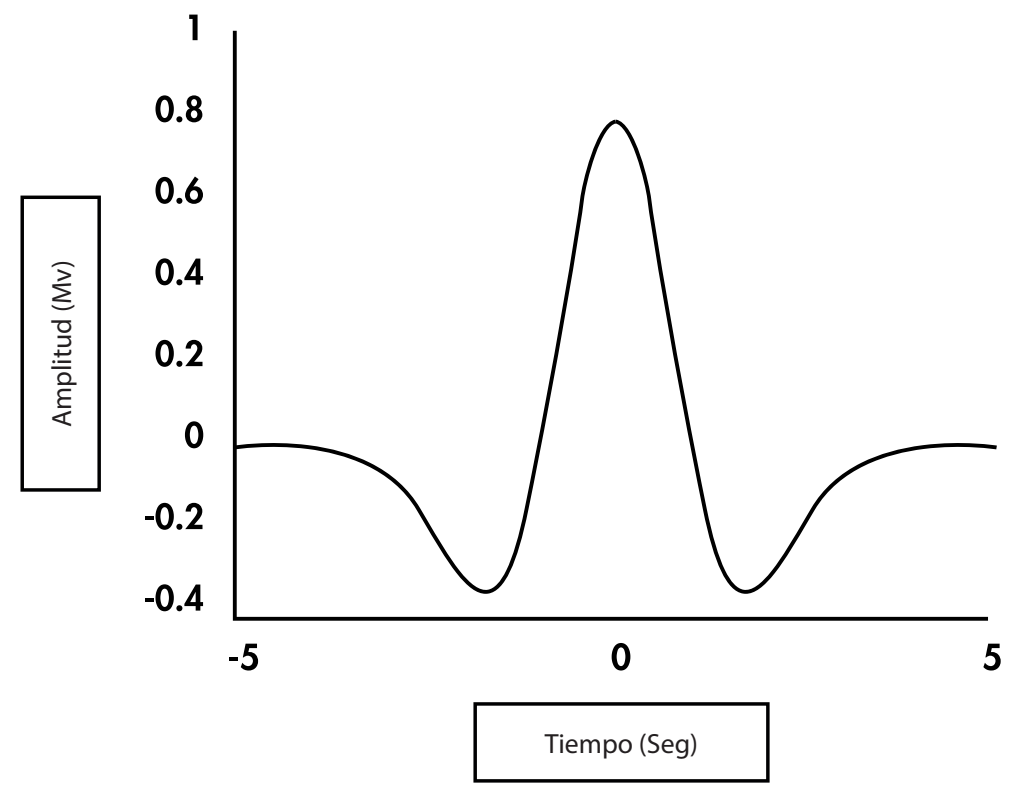

Fuente: elaboración propia

Al haber escogido la función wavelet, se procede a generar una familia de funciones que genere una serie de desplazamientos y escalas en el dominio de la frecuencia (ver ecuación 2).

$$
\varphi_{u, s}=\frac{1}{\sqrt{s}}\left(\frac{t-u}{s}\right)
$$

Se puede generar en Matlab una simulación de esta función en el dominio del tiempo para diferentes escalas de frecuencia de la función sombrero mexicano:

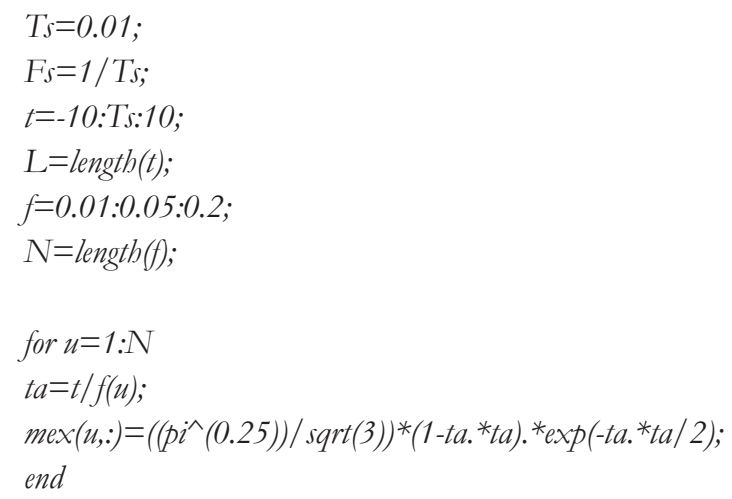

figure

mesh $(t, f$, mex $)$ 
La figura 10 contiene el resultado del proceso de escalamiento de la función Sombrero Mexicano.

Figura 10. Función Sombrero México escalada

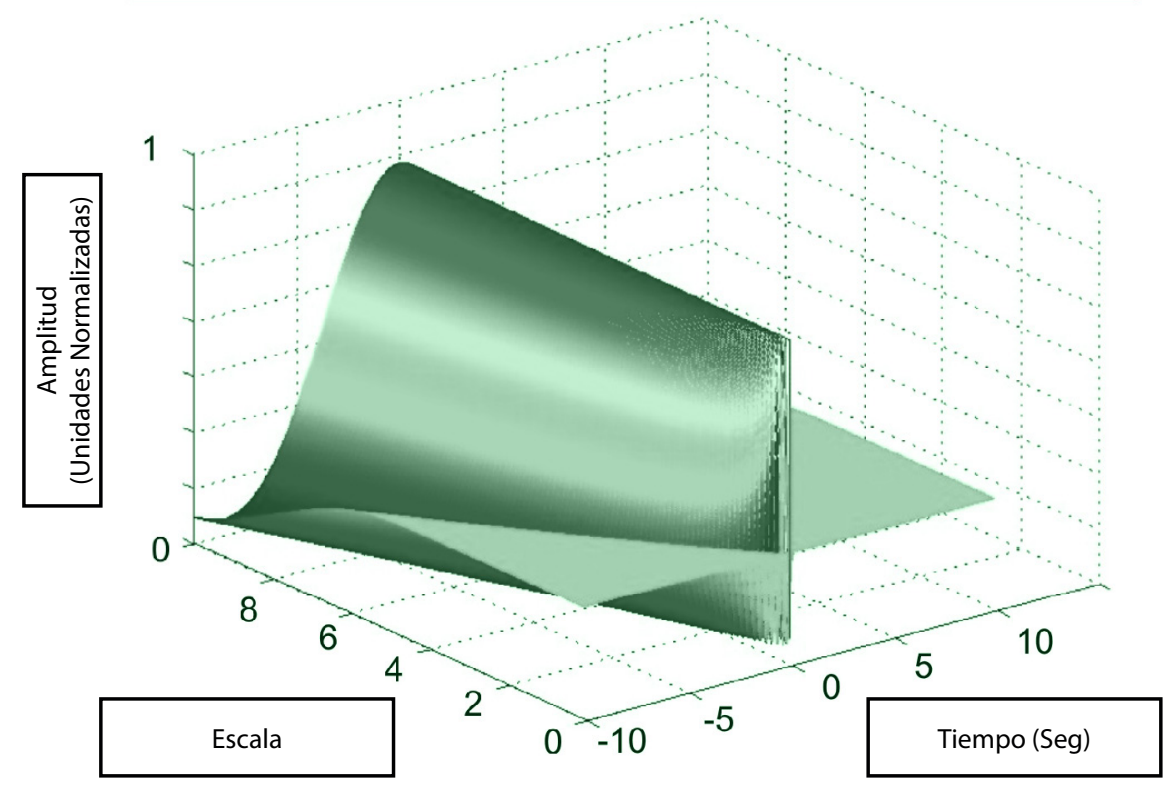

Fuente: elaboración propia

Para ejecutar el análisis basado en la Transformada Wavelet se recure a la ecuación 3. Esta expresión permite que cada una de las versiones escaladas de la función wavelet se opere sobre la función de entrada. La respuesta de esta operación proporciona una función bidimensional con dos ejes: tiempo y frecuencia (escala).

$$
W F(u, s)=\int_{-\infty}^{+\infty} x(t) \frac{1}{\sqrt{s}} \varphi\left(\frac{t-u}{s}\right) d t
$$

A través del siguiente código en Matlab se implementa el proceso de comparación utilizando el comando filter:

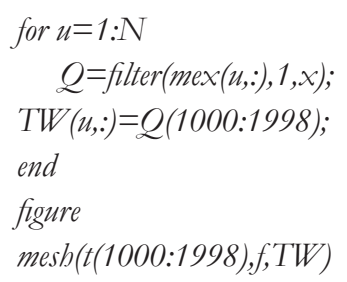




\section{Algoritmo para realce de la onda $\mathrm{R}$ del electrocardiograma}

El resultado obtenido está contenido en la figura 11. Se puede apreciar los picos de amplitud que aparecen en fase con las ondas $\mathrm{R}$ de una señal electrocardiográfica. Esto es producto de la correlación entre la función wavelet Sombrero Mexicano y el complejo QRS de la señal electrocardiográfica.

Figura 11. Transformada Wavelet de una señal electrocardiográfica

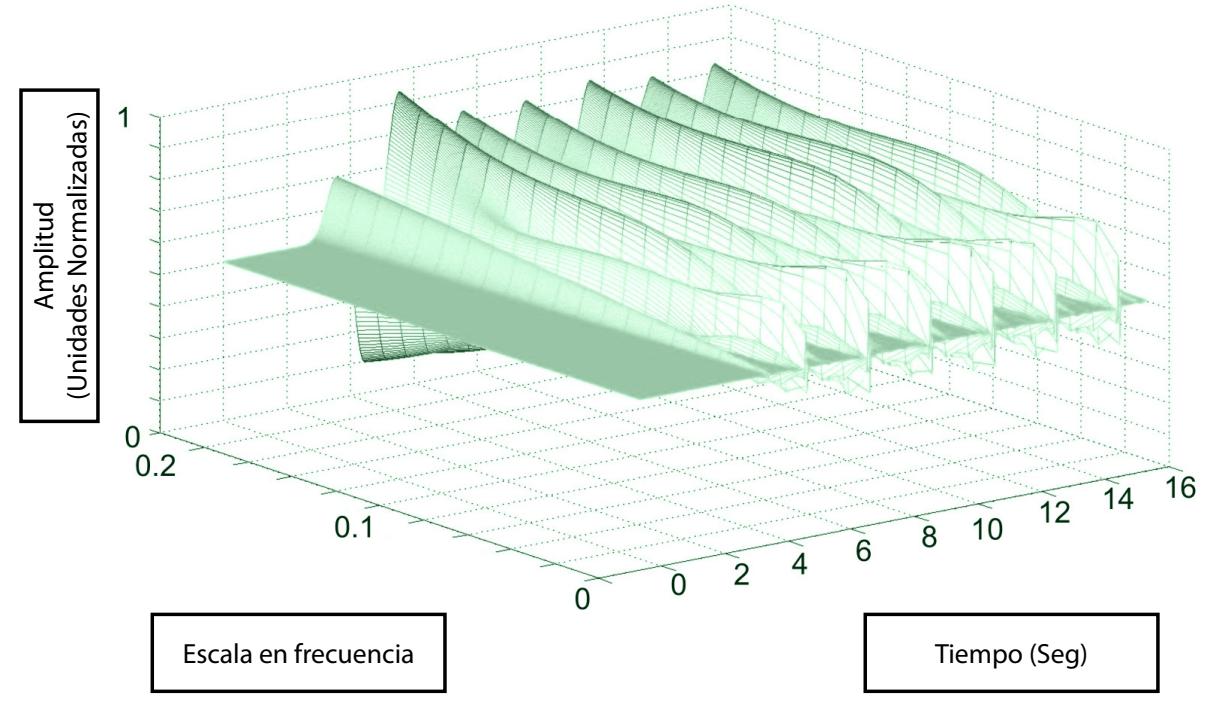

Fuente: elaboración propia

\section{- El proceso de umbralización}

$\mathrm{Al}$ ejecutar un proceso de detección o realce de la onda $\mathrm{R}$ del complejo cardiaco no se obtiene un resultado final. El paso posterior consiste en implementar una serie de pasos propuestos por el autor Tompkins W (1993).

Tompkins sugiere, en primera instancia, utilizar un filtro pasa banda con frecuencias de cortes centradas en $12 \mathrm{~Hz}$ para obtener las componentes asociadas a la onda R. La figura 12 ilustra el resultado obtenido al utilizar una señal ECG tomada del repositorio Physionet (s.f). 


\section{Escenarios de la formación investigativa}

Figura 12. Filtrado pasa banda con frecuencia central de $12 \mathrm{~Hz}$

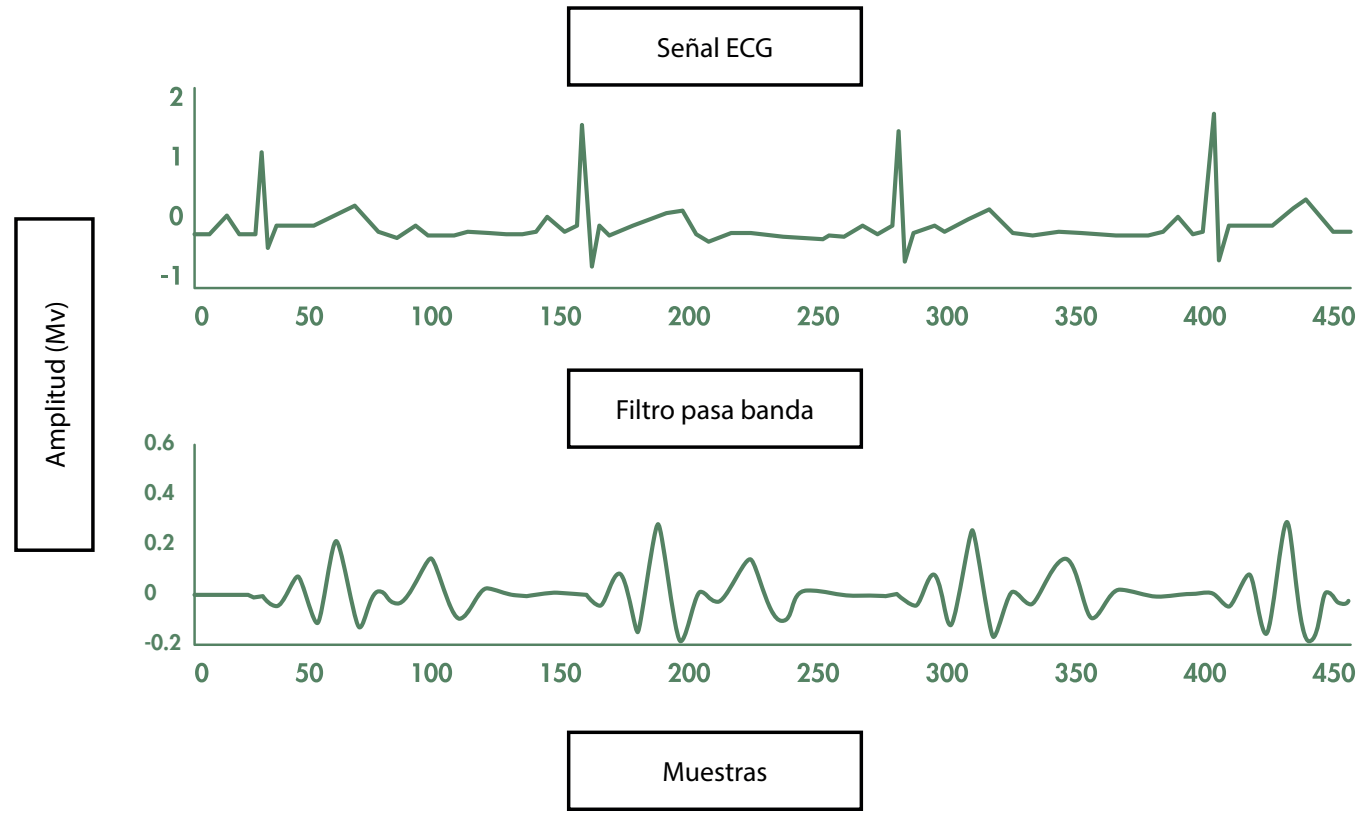

Fuente: elaboración propia

El siguiente paso propuesto consiste en aplicar una serie de filtros derivadores: primera y segunda derivada. El procedimiento tiene como entrada la variable ec que contiene la señal electrocardiográfica. Como salidas se obtienen dos señales: D1 y D2, que corresponden a la primera y segunda derivada de la variable ec.

Este procedimiento se implementa a través de las siguientes líneas de código:

$$
\begin{aligned}
& B O=\left[\begin{array}{lll}
1 & 0 & -1
\end{array}\right] ; \\
& \text { D1 =filter (B0, [1],ec); } \\
& \text { D1 = abs(D1); } \\
& B 1=\left[\begin{array}{llll}
1 & -2 & 0 & 1
\end{array}\right] \text {; } \\
& \text { D2=filter }(B 1,[1], e c) \text {; } \\
& \text { D2=abs(D2); }
\end{aligned}
$$

La figura 13 permite ver en el dominio del tiempo el resultado al calcular la primera y segunda derivada de la señal electrocardiográfica. 
Figura 13. Primera y segunda derivada de una señal ECG

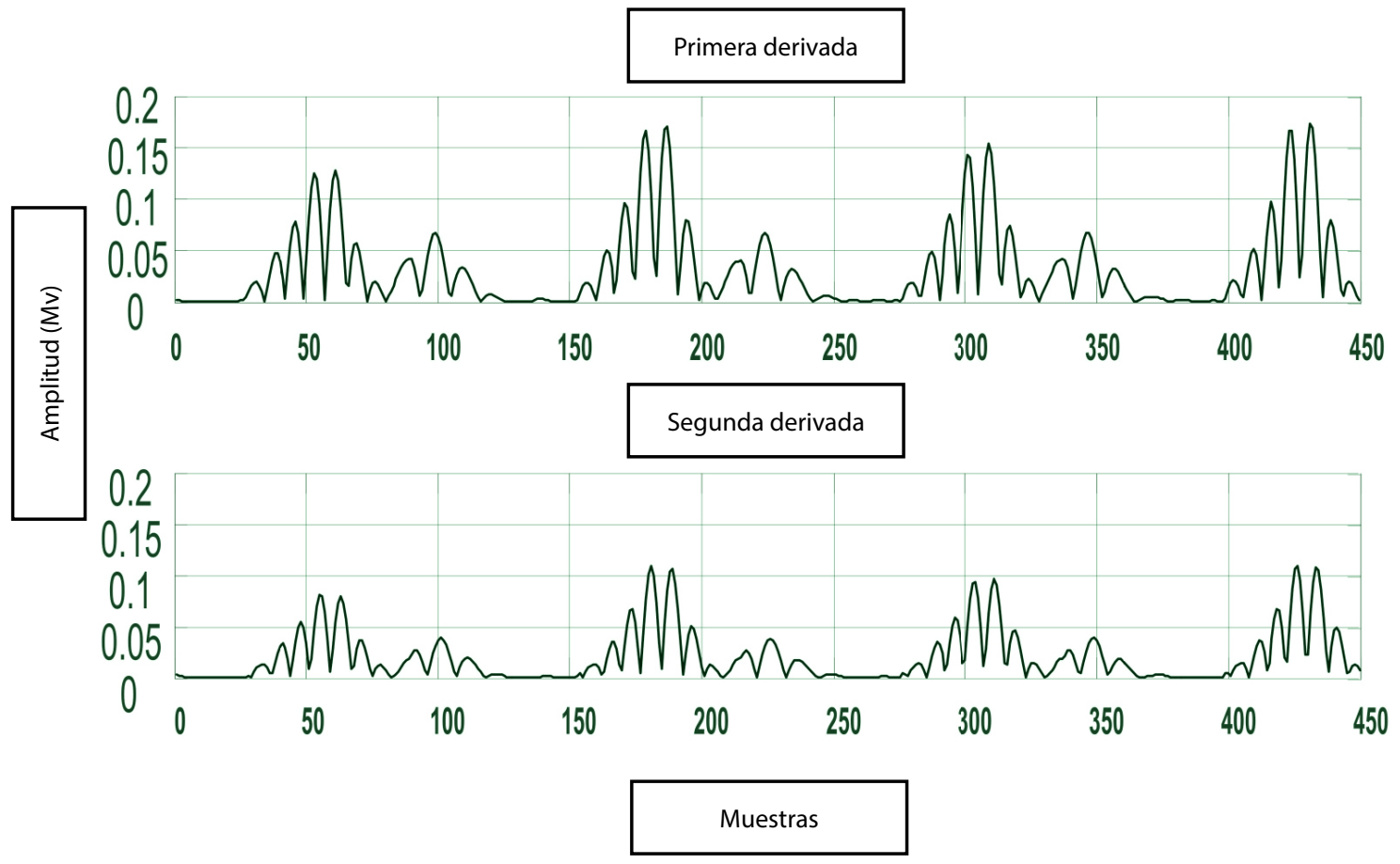

Fuente: elaboración propia

$\mathrm{Al}$ tomar la señal que equivale a la primera derivada (D1) y la equivalente a la segunda derivada (D2) se procede a realizar el cálculo de la nueva señal contenida en la variable $\mathrm{Y}$.

$$
y 2=1.3 * D 1+1.1 * D 2 ;
$$

El resultado obtenido al sumar las operaciones de diferenciación se puede observar en la figura 14. 
Escenarios de la formación investigativa

Figura 14. Suma de primera y segunda derivada de un ECG

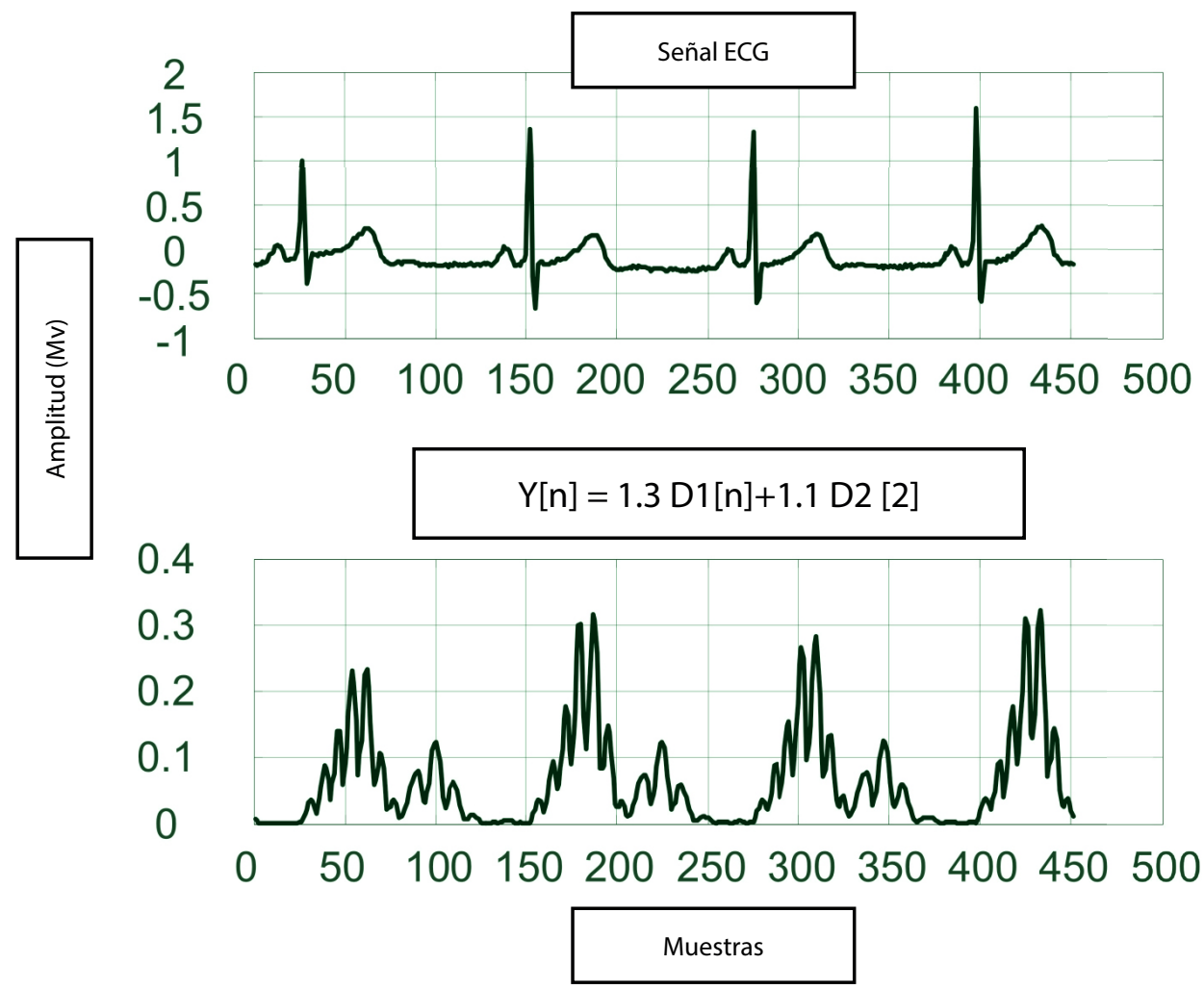

Fuente: elaboración propia

Por último se procede a un proceso de suavizado utilizando un filtro promediador o integrador. El código para implementar el proceso de suavizado es el siguiente:

$$
\begin{aligned}
& L=16 ; \\
& B p=(1 / L) * \text { ones }(1, L) \\
& y p=\text { filter }(B p, 1, y) ;
\end{aligned}
$$

El proceso de suavizado genera la variable yp que corresponde a una versión de la señal ECG en la cual el pico de mayor amplitud indica la presencia de una onda $\mathrm{R}$ (ver figura 15). 
Figura 15. Proceso de promediado

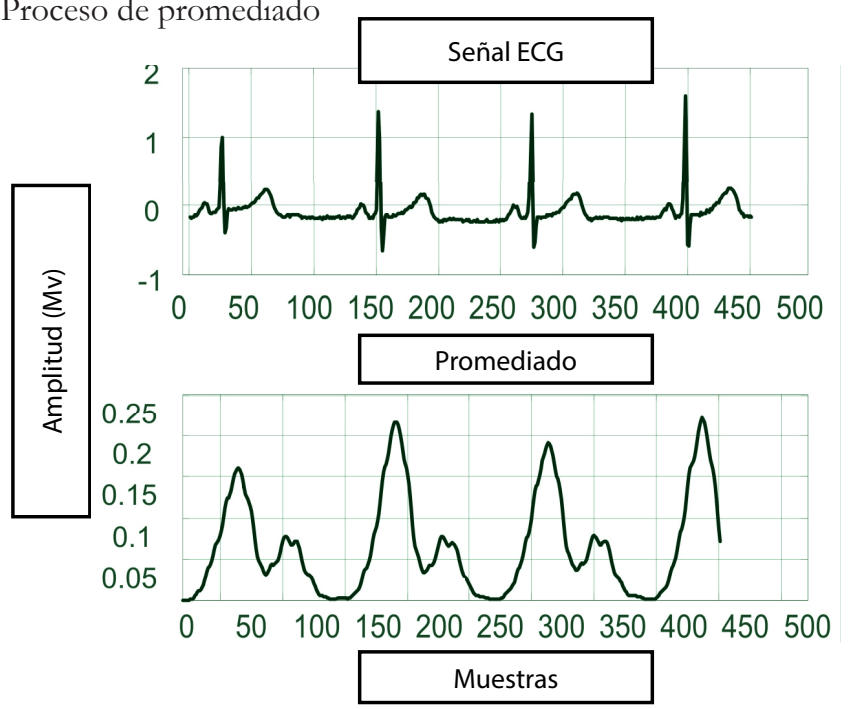

Fuente: elaboración propia

Para poder cuantificar el tiempo entre cada onda R se procede a la aplicación de un umbral a la señal promediada. En el siguiente código se realiza una operación de normalización a la variable yp, con la finalidad de obtener una señal con solo valores entre 0 y 1 . Posteriormente se aplica un operador de umbral:

$$
\begin{aligned}
& M=\max (y p) ; \\
& y p=y p / M ; \\
& y u=y p>0.6 ;
\end{aligned}
$$

El resultado obtenido en el proceso de umbralización está contenido en la figura 16.

Figura 16. Umbralización de la señal ECG

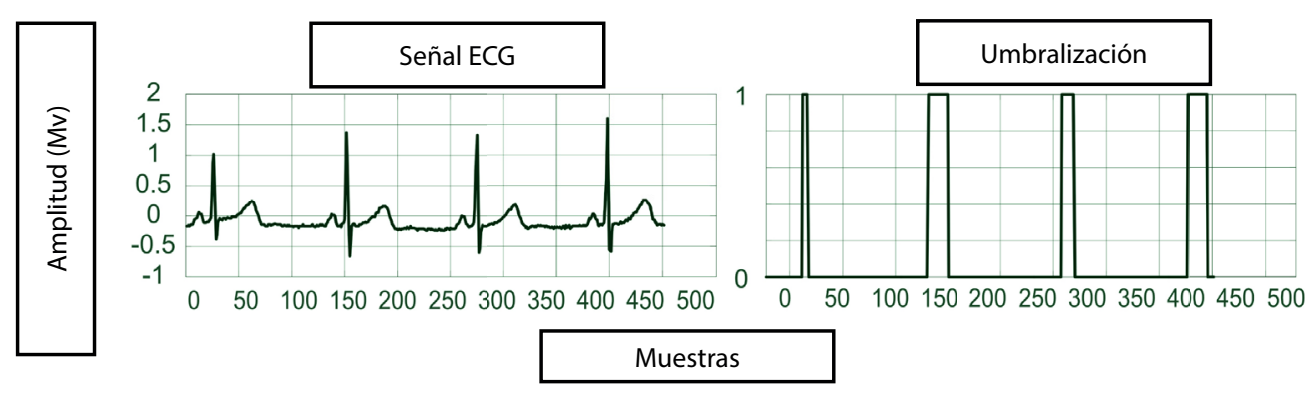

Fuente: elaboración propia 


\section{Escenarios de la formación investigativa}

Los algoritmos para la umbralización de la frecuencia cardiaca han sido complementados con diferentes técnicas. Como ejemplo se tiene el caso del uso de herramientas estadísticas como el histograma, que permite establecer un umbral adaptativo basado en el comportamiento de los valores de amplitud de la señal ECG adquirida (González-Barajas, Velandia-Cardenas y Nieto-Camacho, 2015).

\section{- La Señal de Ritmo Cardiaco}

La señal de ritmo cardiaco es una serie de tiempo que contiene la información de los intervalos entre cada onda $\mathrm{R}$ del complejo cardiaco. Las unidades de este tipo de señal están en segundos y corresponde al tiempo transcurrido entre cada una de las ondas. El inverso matemático de estos valores corresponde al término: Frecuencia cardiaca instantánea.

La señal de ritmo cardiaco se puede extraer a partir del proceso de umbralización ilustrado en la figura 16 cuyo resultado es un tren de impulsos de valor unitario que representan cada una de las ondas $\mathrm{R}$.

Para realizar el cálculo de dicha señal es necesario seguir algunos pasos:

1 Se toma la variable yu que contiene la señal umbralizada y se procede con la instrucción find a establecer la ubicación de cada muestra de la señal que posee un valor igual a uno.

$\%$ arreglo con $\mathrm{N}^{\circ}$ de posiciones de los valores iguales a 1 :

$y 1=f i n d(y u==1)$;

Como se pudo observar en la anterior línea de código, la variable y1 corresponde a un arreglo de datos que contiene las posiciones con valor igual a uno.

2 Se procede a estimar las posiciones iniciales de los trenes de impulsos. Este procedimiento se realiza con el fin de establecer el inicio de una onda R. Las siguientes líneas de código toman la variable y1 para originar los arreglos y1a y y1b, para generar la variable y2:

$$
\begin{aligned}
& y 1 a=\left[\begin{array}{ll}
y 1 & 0
\end{array}\right] ; \\
& y 1 b=\left[\begin{array}{ll}
0 & y 1
\end{array}\right] ; \\
& y 2=y 1 a-y 1 b ;
\end{aligned}
$$

La variable y 2 permite establecer la distancia en muestras entre cada posición que contiene un valor unitario. Cuando existen dos posiciones consecutivas, la variable y2 tomará el valor de uno.

En el caso que existan dos posiciones lejanas, la variable y2 tendrá un valor ma- 


\section{Algoritmo para realce de la onda $\mathrm{R}$ del electrocardiograma}

yor de uno. Este escenario se debe interpretar como el hallazgo de un nuevo tren de impulsos, el cual indica el inicio de una nueva onda R.

$3 \mathrm{Al}$ tener caracterizada la señal se deben extraer las posiciones de inicio del tren de impulsos, lo cual se puede realizar a través de la siguiente instrucción:

$$
p=\operatorname{find}(y 2>1)
$$

4 Se procede a calcular la cantidad de muestras entre cada inicio de los trenes de impulsos. A través de las siguientes líneas de código se genera la variable pos, que contiene las posiciones que indican el inicio de cada tren de impulsos:

$$
\begin{aligned}
& L 1=\text { length }(p) ; \\
& \text { for }=1: \text { L1 } \\
& C=p(i) \text {; } \\
& \operatorname{pos}(i)=y 1(C) \text {; } \\
& \text { end } \\
& \text { pos } 1=[\operatorname{pos} 0] \text {; } \\
& \text { pos } 2=\left[\begin{array}{l}
0 \\
\text { pos }
\end{array}\right] \text {; } \\
& \text { pos3 }=\text { pos1-pos } 2 \text {; } \\
& \text { pos4=pos3(1:L1); }
\end{aligned}
$$

La variable pos4 contiene la cantidad de muestras entre cada inicio de tren de impulsos. Esto es equivalente a la distancia entre cada onda $\mathrm{R}$ del complejo cardiaco.

5 Para obtener el valor de la distancia en unidades de tiempo se debe multiplicar cada valor de la variable pos4 por el periodo de muestreo Ts:

$$
\begin{aligned}
& T s=0.008 ; \\
& \text { tiempo }=\text { pos } 4 * T s ;
\end{aligned}
$$

La variable tiempo contiene, en unidades de segundos, la distancia entre cada onda R. Este arreglo de datos contiene los valores denominados: Tiempos R-R (Ver figura 17). Estos valores son el componente de la señal de ritmo cardiaco y son la base de estudios para el análisis de la variabilidad de la frecuencia cardiaca. 
Figura 17. Señal de ritmo Cardiaco
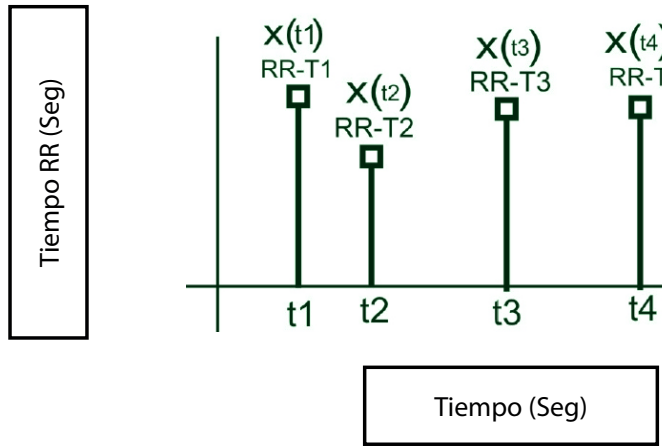

Fuente: elaboración propia

\section{- Frecuencia cardiaca instantánea}

Finalmente una vez calculada la señal de ritmo cardiaco basta con calcular su inverso para poder obtener la gráfica de la frecuencia cardiaca instantánea. Esta gráfica se puede obtener por medio de las siguientes líneas de código:

\section{$V F R=1 . /$ tiempo;}

El resultado de dicha operación proporciona un arreglo de datos almacenado en la variable VFR que contiene el inverso matemático de los datos expuestos en la figura 14. Estos nuevos datos tienen una escala de frecuencia en hertzios $(\mathrm{Hz}=1 / \mathrm{s})$.

El análisis de la señal de ritmo cardiaco ha requerido herramientas basadas en índices estadísticos (dominio del tiempo) y la Transformada de Fourier (dominio de la frecuencia). Como aporte al estado del arte se han logrado estrategias para mejorar el análisis en el dominio de la frecuencia aplicado a datos irregularmente muestreados (González-Barajas, Francisco-Forero y Marino-Martinez, 2014b). 


\section{Análisis de resultados}

Los algoritmos propuestos en este capítulo pueden ser probados de diferentes maneras. Para lograr los resultados expuestos en cada numeral fue tomado como escenario de prueba las señales de la base de datos Physionet (s.f).

Las señales utilizadas fueron tomadas de la sección de Señales de Ritmo Sinusal Normal (MIT- BIH Normal SinusRhythm Data Base). Se tomaron al azar un número de diez registros electrocardiográficos con frecuencia de muestro Fs= 333.333 muestras/ segundo y ventana de 2000 muestras. En promedio, cada ventana posee 10 ondas $\mathrm{R}$ para detectar.

En este capítulo se exponen dos puntos de vista para detectar la onda R: Transformada Wavelet Continua y Bancos de Filtros. Se realizaron varias pruebas para comparar la cantidad de ondas R que cada algoritmo podría detectar correctamente. En la tabla 1 se documenta una comparación entre los algoritmos basados en Transformada Wavelet Continua y Bancos de Filtros.

Tabla 1.

\begin{tabular}{|c|c|c|}
\hline Estrategia & $\begin{array}{c}\text { cantidad de picos } \\
\text { de señal }\end{array}$ & Picos detectados \\
\hline $\begin{array}{c}\text { Transformada } \\
\text { Wavelet continua }\end{array}$ & 7 & 5 \\
\hline Banco de filtros & 7 & 6 \\
\hline
\end{tabular}

Fuente: elaboración propia

Como se pudo ver en la tabla 1 existe la posibilidad de que la estrategia basada en Bancos de Filtros pueda generar un mayor número de ondas $\mathrm{R}$ detectadas. También se debe tener en cuenta que la estrategia Bancos de Filtros permite generar un rango de frecuencias para la implementación de un sistema de filtrado digital. La Transformada Wavelet Continua requiere una arquitectura más compleja para detectar la onda R. 
Este resultado nos da una base muy sólida para continuar con la investigación y proponer sistemas más robustos para el análisis del ECG. Los nuevos resultados proporcionarán ideas para el diseño desistemas capaces de tomar y procesar de forma óptima muestras correspondientes a los estándares mundiales (que hablan de exámenes que van desde los 5 minutos hasta las 24 horas seguidas), asegurando un mínimo porcentaje de error. Estos sistemas incluirán también el cálculo de los parámetros en el dominio del tiempo y de la frecuencia con los que se analizan la señal de ritmo cardíaco y la frecuencia cardiaca instantánea. Estos parámetros hacen posible un análisis mucho más profundo del estado del corazón, generando reportes numéricos sobre su funcionamiento. 


\section{$\mid$ conclusiones $\mid$}

El procesamiento digital de señales es un área de la ingeniería que ha permitido generar estrategias para extraer información de las señales electrofisiológicas. Estos aportes han sido utilizados para dos fines específicos: atenuar distorsiones y permitir la detección de ondas características de la señal bajo estudio.

El paso posterior al proceso de obtener una señal ECG libre de ruidos consiste en implementar estrategias para la detección de la onda R. Los resultados expuestos en este capítulo permiten analizar el desempeño de la aplicación de bancos de filtros para encontrar un rango de frecuencias con las componentes espectrales asociadas a la onda $\mathrm{R}$. Al poder realizar la detección de la onda $\mathrm{R}$ se obtiene la posibilidad de construir la señal de Ritmo Cardiaco. Así mismo al aplicar métodos como la transformada wavelet es posible, a partir de patrones, distinguir y acentuar ciertos componentes de las señales posibilitando realizar análisis más profundos y precisos, sentando así otra opción para resaltar la onda $\mathrm{R}$. 


\section{Referencias}

Barrero, J., González, J. y Pinto, M. (2011). Cardiotacómetro Digital Prototipo para Estudio de la V de la Frecuencia Cardiaca (pp. 81-86). Revista UIS Ingenierías, vol. 2, no 2.

Enderle, J. y Blanchard, S. (2000). Introduction to Biomedical Engineering (p. 193). USA. Academic Press Ed.

González-Barajas, J. (2014a).Threshold calculation for $\mathrm{R}$ wave detection in complex cardiac, 17(32), 47-55.Tecno Lógicas.

González-Barajas, J., Velandia-Cárdenas, C., y Nieto-Camacho, J. (2015). Implementación de filtro digital en tiempo real para detección de la onda R, 18(34), 75-86. Tecno Lógicas.

González-Barajas, J., Francisco-Forero, E. y Marino-Martinez, I. (2014b). Tratamiento de rizados en la estimación de la densidad espectral de potencia de la señal de ritmo cardiaco. REVISTA UIS INGENIERÍAS, 12(2).

González-Barajas, J., y Montenegro, D. (2014c). Digital analysis and treatment for electromagnetic disturbances in biomedical instrumentation. OmniaScienceMonographs.

Guyton, A. y Hall, J. (2011). Tratado de fisiología médica (p. 105). Duodécima edición. España: GEA consultoría editorial, S.L..

Koeppen, B. y Stanton, B. (2009). Fisiología (p. 292).Barcelona, España: ElseveierMosbe Ed.

López, J. y Fernández, A. (2008). Fisiología del ejercicio (pp. 321-340). Bogotá, Colombia: Panamericana ed. Physionet (s.f). Recuperado de http://www.physionet.org/cgi-bin/atm/ATM?database=nstdb\&tool=plot_waveforms

Tompkins, W. (1993).Biomedical Digital Signal Processing (p. 43).New Jersey, USA: Prentice Hall. 


\section{Consejo Editorial}

Fr. Juan Ubaldo López Salamanca, 0. P. Rector General

Fray Érico Juan Macchi Céspedes, O.P.

Vicerrector Académico General

Fray Diego Orlando Serna, 0. P.

Vicerrector Administrativo y Financiero

General

Fray Tiberio Polanía Ramírez, O.P.

Vicerrector General de la Universidad Abierta y a Distancia (VUAD)

\section{Unidad de Investigación}

PhD. Henry Borja Orozco

Director

Camila Suárez

Docente Investigadora

Coordinadora del programa de jóvenes

investigadores

\section{Comité de Selección}

Mg. Beatriz Mena Bejarano

Universidad Nacional de Colombia

PhD. Mario Solarte Rodríguez

Pontificia Universidad Javeriana

PhD. Amanda Romero Medina

Universidad Pedagógica Nacional

Mg. Rodrigo Alvarado Escobar

Universidad Sergio Arboleda

PhD. José Manuel Salamanca Rangel

Pontificia Universidad Javeriana

Mg. Andrea Juliana Enciso

Universidad de Pittsburgh
Esp. Adriana Jiménez Herrera

Secretaría de Educación de Bogotá

Mg. Claudia Carmona Rodríguez

Universidad Pontificia Bolivariana

Mg. Natalia Escobar Sabogal

Universidad de los Andes

PhD. Andrés García-Suaza

Colegio Mayor de Nuestra Señora del Rosario

Mg. Franklin Púa Mora

Universidad de San Buenaventura

Mg. Paola Riaño Arbeláez

Universidad Militar Nueva Granada

Esp. Catalina Castro Gaitán

Publicaciones Semana

Mg. Edwin Gamboa

Universidad Sergio Arboleda

PhD. Luz Amalia Hoyos Cuartas

Universidad Pedagógica Nacional

PhD. Sonia Helena Contreras

Universidad Tecnológica de Bolivar

PhD. Diana Paola Guzmán

Universidad Jorge Tadeo Lozano

Mg. Andrés Camilo Torres Estrada

Universidad de los Andes

Mg. José Arturo Ángel Poveda

SED Bogotá y Compensar

Mg. Claudia Padrón Mercado

Fundación Universitaria Konrad Lorenz 
Esta obra se editó en Ediciones USTA, Departamento Editorial de la Universidad Santo Tomás. Se usó papel esmaltado de 300 gramos para la carátula y papel papel book cream de 59.2 gramos para páginas internas. Tipografía: Garamond y Futura. Impreso por Xpress 
Los semilleros de investigación en la Universidad Santo Tomás se han venido configurando alrededor de la idea de que las habilidades de investigación se adquieren solo en el ejercicio y la práctica investigativa. A esto se refiere el principio que fundamenta la conformación de estos colectivos: "aprender a investigar investigando”. Es por esto que desde el 2010, la Universidad ha hecho esfuerzos importantes para fomentar y consolidar a los semilleros de investigación como una de las estrategias institucionales más importantes en lo que a formación investigativa se refiere.

La Colección Semillas ha sido creada con el propósito de ampliar los escenarios de visibilización de la investigación hecha por los semilleros de la Universidad. En este libro confluyen los esfuerzos institucionales por incentivar la producción investigativa de los estudiantes, que con el paso del tiempo han venido incrementando en número y en calidad su participación en la generación de productos que, además de fortalecer a los grupos de investigación, contribuyen al posicionamiento y reconocimiento en el ámbito nacional e internacional de nuestra institución. 